\title{
Sébastien Fleuret et Anne-Cécile Hoyez, Santé et géographie. Nouveaux regards
}

David Lessault

\section{(2) OpenEdition}

Édition électronique

URL : https://journals.openedition.org/remi/6446

DOI : $10.4000 /$ remi.6446

ISSN : $1777-5418$

Éditeur

Université de Poitiers

Édition imprimée

Date de publication : 1 juin 2013

Pagination : 138-140

ISBN : 979-10-90426-08-5

ISSN : 0765-0752

\section{Référence électronique}

David Lessault, « Sébastien Fleuret et Anne-Cécile Hoyez, Santé et géographie. Nouveaux regards », Revue européenne des migrations internationales [En ligne], vol. 29 - n² | 2013, mis en ligne le 01 juin 2013, consulté le 15 avril 2022. URL : http://journals.openedition.org/remi/6446 ; DOI : https://doi.org/ 10.4000/remi.6446 


\section{Notes de lecture}

Bashi, Vilna Francine

Survival of the Knitted. Immigrant Social

Networks in a Stratified World. - California:

Stanford University Press, 2007. - 319 p.

ISBN : 978-0-8047-4090-6

L'ouvrage traite des réseaux sociaux des immigrés en montrant comment ils peuvent être un outil de mobilité sociale. L'auteure étudie le cas d'immigrants résidant dans les zones métropolitaines de Londres et de New York et de leurs familles et amis restés dans leurs îles d'origine de la Caraïbe-Est, anglophone. Elle entend montrer que le réseau joue un rôle essentiel dans la mesure où il permet à l'immigrant de tisser et d'entretenir des liens pour mieux vivre dans un milieu étranger, voire hostile.

Dans le premier chapitre, qui débute par le récit d'une enseignante originaire de Trinidad, sur les raisons de sa migration au Royaume-Uni, Bashi annonce sa démarche méthodologique : décoder le sens des pratiques dont les migrants rendent compte dans leurs récits. Mais l'auteure situe aussi ses recherches dans le contexte international où les réseaux sociaux d'immigrants opèrent (chapitre 2). Elle montre comment les destinations sont choisies et décrit les situations politique, économique et raciale que rencontrent ces immigrants issus majoritairement de milieux modestes. Le troisième chapitre décrit les réseaux de migrants caribéens de manière détaillée et montre comment se pratique la "sélection " des immigrants dans la perspective de leur insertion dans des niches spécifiques sur le marché du travail. Bashi examine la structure du réseau, au cœur du système socioéconomique du migrant, en mettant l'accent sur une différence fonctionnelle entre deux catégories de migrants désignées respectivement sous les termes de " hub " et
" spoke ". Le " hub " qualifie celui qui fait venir des compatriotes, de manière légale ou illégale, dans le pays d'accueil. Le "spoke " est celui, qui bénéficie de l'assistance du « hub ». En s'appuyant sur des entretiens menés avec des Caribéens (" West Indians ") restés dans la Caraïbe et avec des migrants à New York et à Londres ou dans ses environs, elle relève cinq caractéristiques propres au fonctionnement du "hub ": lien avec le pays d'origine, capacité à se mettre au service de la communauté, culture de la réciprocité, position clé sur le marché du travail et sélection des migrants.

Dans les chapitres 4 et 5, Bashi discute la notion de culture de la réciprocité en reprenant partiellement les propositions de Thomas Faist ${ }^{1}$ concernant deux éléments essentiels au fonctionnement du réseau : le capital social et la confiance. Le capital social est considéré comme un ensemble de ressources relationnelles dont l'accès dépend de l'appartenance au réseau. II existe ainsi des groupes de parenté (" kinship groups ") qui tissent des liens de réciprocité et organisent des circuits transnationaux (" transnational circuits ") basés sur l'échange. Toutefois, l'auteure se démarque quelque peu de ces approches en mettant l'accent sur le rôle clé des " hubs", véritables détenteurs du capital social dans les réseaux immigrés, soulignant par là même leur considérable influence dans la sélection des nouveaux arrivés qui bénéficieront ensuite des ressources et des connexions utiles.

1 Faist Thomas (1997) The Crucial

Meso Level, in Thomas Hammar Ed., International Migration, Immobility and Development: Multidisciplinary Perspectives, Oxford, Berg, pp. 47-64. 
Les chapitres suivants (6 et 7) rendent compte de l'utilité réelle du réseau, en particulier dans une société où de nombreux obstacles et discriminations affectent le parcours de ces immigrés. Mais la seule appartenance au réseau ne suffit pas pour atteindre la mobilité sociale souhaitée et s'il est un moyen d'égaliser en partie les conditions de vie ou de mieux répartir les bénéfices et avantages dans des espaces socioéconomiques hiérarchisés, il peut être aussi générateur d'inégalités. Dans le huitième et dernier chapitre, l'auteure met en évidence ses limites dans des contextes où des politiques migratoires sélectives privilégient souvent ceux qui ont un meilleur bagage socioéconomique et intellectuel.

Cet ouvrage, pour conclure, fournit des éléments de connaissance précieux sur la constitution des réseaux de migrants caribéens notamment dans l'usage que fait Bashi du modèle " hub et spoke " que I'on retrouve dans nombre de processus migratoire. Mais ce modèle ne mériterait-il pas d'être nuancé, alors que l'accélération des moyens de communication permet au nouveau migrant d'accéder plus rapidement aux informations nécessaires, notamment en matière d'emploi, le rendant ainsi plus autonome ? La fonction $\mathrm{du}$ " hub ", intermédiaire que l'auteure nous présente comme central, n'est-elle pas à questionner à nouveau dans cette logique?

Clara Rachel Eybalin Casséus Doctorante Université de Poitiers (MIGRINTER)
Fleuret, Sébastien (dir.)

Hoyez, Anne-Cécile (dir.)

Santé et géographie. Nouveaux regards. -

Paris : Economica, Anthropos, 2011. - 302 p. ISBN : 978-2-7178-6098-6

Tous les deux géographes au laboratoire Espace et Sociétés (ESO) du CNRS, S. Fleuret et A.-C. Hoyez sont spécialistes des questions de santé. Les textes qu'ils rassemblent dans ce volume ont vocation à se démarquer des travaux classiques et " occidentalo-centrés " de la géographie de la santé en éclairant des domaines d'investigation moins exposés. $\mathrm{Et}$, pour sortir des " sentiers battus ", les auteurs nous dévoilent un panorama d'études géographiques "qui vont voir ailleurs, qui voient d'ailleurs, qui parlent d'ailleurs, que ce soit en termes de lieux, de pensée, de groupes étudiés ". Ce choix entend ainsi compléter utilement une première compilation de textes publiés dans la même collection cinq ans plus tôt (Fleuret et Thouez, 2007). Et c'est chose réussie, au fil de onze chapitres nous accompagnant de I'Inde au Brésil, de l'Afrique en Europe dans un dialogue franco-britannique et inter-disciplinaire critique sur les approches classiques d'une question universelle dont les pratiques s'inscrivent dans des milieux socio-culturels si variés que la " spatialisation du regard " est plus qu'une invitation, une nécessité.

Les onze chapitres de l'ouvrage sont distribués en deux parties distinctes : " $\mathrm{La}$ première suggère un autre regard sur le monde ". II s'agit d'abord de porter un regard critique sur la mondialisation et ses enjeux dans le domaine de la santé. Le premier chapitre est conçu comme un dialogue franco-britannique sur la base des travaux d'A. Vaguet et de S. Atkinson menés en grande partie dans des pays du Sud. Dans le chapitre suivant, T. Brown propose une lecture critique du concept de santé publique mondiale ou encore " globale ". Son point de vue est complété, appuyé par les propos défendus dans le texte suivant par deux autres chercheurs 
N. Warfa et A.-C. Hoyez qui, mobilisant leurs expériences de terrain menées en Inde et en Afrique, donnent leur point de vue sur les modèles biomédicaux en place dans ces deux contextes particuliers. Le quatrième chapitre achève la première partie en proposant une lecture croisée, celle de deux chercheurs canadiens et celle de deux chercheurs français, sur la construction progressive de la " géographie de la santé " comme discipline à part entière.

On comprend ainsi pourquoi la géographie de la santé a sa propre épistémologie, comment elle s'est écrite et se renouvelle aujourd'hui " au gré des évolutions et des changements dans la façon de se saisir des problématiques spatiales issues de la géographie (diffusion, réseaux, mobilités, accès...) et des problématiques sociales replaçant la santé comme fait social global, c'est-à-dire touchant à tous les aspects de la vie en société, des phénomènes macro aux micro ". La "discipline ", de laquelle les auteurs se réclament, est ainsi marquée, écrivent-ils, "par les réflexions ayant conduit à l'adaptation et la mise en place d'outils communs à la géographie quantitative (SIG, SMA) et qualitative (outils de l'ethnographie appliqués à la géographie) et/ou spécifiques à la géographie de la santé ".

La deuxième partie de l'ouvrage compile une série de textes se démarquant des principaux courants en géographie de la santé pour faire place aux travaux consacrés d'une part aux populations peu étudiées et $d$ 'autre part à des formes alternatives d'organisation de l'offre de la santé. Ainsi V. Chasles propose une analyse des implications de l'effet de genre dans les mobilités de santé en contexte indien. C. Richmond et K. Wilson s'intéressent au cas aborigène dont les valeurs fondatrices de la conception de la santé sont mises à mal par les évolutions du monde moderne. C'est ensuite, les travaux d'un groupe de chercheurs franco-britanniques qui sont présentés. Ils traitent des interactions entre migration et santé en France et en Grande Bretagne en privilégiant une approche politique, sociale et culturelle. Les quatre derniers chapitres sont consacrés à des formes originales de structuration de l'offre de santé. L'évolution récente des systèmes de santé, roumain et brésilien, est abordée dans deux chapitres. Dans le cas roumain, traité par E. Bioteau et S. Fleuret, le système de santé post-communiste est transformé par des influences extérieures fortes, orchestrées par les instances internationales se substituant aux dynamiques locales en gestation après une longue période collectiviste. Inversement, M.-G Medina et S. Fleuret montrent, dans le cas brésilien la mise en place de programmes basés sur le diagnostic micro-local et l'attention portée à la participation de la population dans le cadre d'une approche résolument territoriale de la politique de santé primaire. La démarche participative est également au centre de l'analyse d'A. Power qui étudie dans son texte les implications des secteurs communautaires et bénévoles dans les systèmes de santé observés dans divers contextes géographiques. Enfin, G. Andrews, J. Adams et J. Segrott approfondissent, dans le dernier chapitre, l'évolution des " médecines traditionnelles " en " médecines alternatives et complémentaires " (CAM) et leurs relations à l'espace et aux lieux, dans leur diffusion comme dans leur pratique. Ainsi ils concluent que la recherche sur les CAM pourrait contribuer au développement de la géographie de la santé, afin d'étendre les ramifications de cette sous-discipline, en particulier en ce qui concerne les notions de bien-vivre, de bien-être et de bonheur.

Certains géographes pourront sans doute regretter que les apports spécifiques de la discipline ne soient pas toujours clairement mis en avant, en particulier en ce qui concerne la « boîte à outils " mobilisée. Certes, la " spatialisation du regard " dévoile la richesse d'une confrontation des contextes nationaux, des milieux (rural/ urbain), mais le recours à l'analyse spatiale des phénomènes étudiés et à leur repré- 
sentation cartographique est en revanche peu sollicité. Si on perçoit l'intérêt de ces " nouveaux regards " sur la santé on ne peut alors que suivre les auteurs dans leurs intentions de développer d'autres modes d'analyse à partir des systèmes d'information géographiques (SIG) ou encore de suivi longitudinal des populations (données biographiques, etc.).

On sort de cette lecture à la fois dérouté par le voyage que nous proposent les différents contributeurs et méditatifs tant les textes invitent à décentrer le point de vue et engager des pistes de travail nouvelles. Les auteurs nous font finalement partager un besoin convergent d'émancipation par rapport au modèle dominant centré sur une vision " occidentale ", qui fait des questions de santé un objet répondant à des normes qui induit la création de modèles d'action et d'intervention surtout élaborées par et pour des pays du Nord, mais malgré tout transposés dans les pays du Sud. Au fond, ce que les auteurs veulent pointer, c'est bien le risque d'appauvrissement d'une recherche qui se concentrerait sur un nombre restreint de sujets et occulterait les réalités en lien avec des populations minoritaires ou marginalisées (migrants, peuples autochtones, etc.) ou des pratiques " autres " (CAM, recours au tiers secteur). Que la géographie de la santé propose $d^{\prime}$ " autres " visions semble constituer une certaine avancée... dans les domaines de la santé et de la géographie.

David Lessault MIGRINTER CNRS/Université de Poitiers
Patron, Marie-Claire

The legacy of the baby boomers or the french social system? Issues of Equality and Brain Drain. - Rotterdam: Sense Publishers, 2012. 201 p. (Bold visions in educational research) ISBN : 978-9-4620-9069-9

Le dernier ouvrage de Marie-Claire Patron, chercheuse australienne d'origine franco-mauricienne porte sur une question brûlante touchant la société française et son avenir sur la scène internationale. Son livre présente les résultats d'une enquête empirique, menée sur plusieurs années. La chercheuse, spécialiste des questions interculturelles (Patron, 2007 ; 2009), enrichit ici sa réflexion en s'intéressant plus particulièrement aux raisons de la croissance des expatriations des diplômés français, pourtant connus pour leur réticence à ce type de stratégie professionnelle, et à leurs implications sur le système social français.

Cet ouvrage s'articule autour de sept chapitres s'intéressant, tour à tour, aux différents aspects relatifs à l'explicitation de ce mouvement migratoire spécifique. Il débouche sur un chapitre conclusif qui présente les enjeux d'une situation encore bien peu explorée par les chercheurs français (Philip, 2007).

Chercher à saisir l'expatriation d'un type particulier de population, les Français diplômés de l'enseignement supérieur, est un pari ambitieux et difficile tant la variété des situations a rendu l'analyse délicate (chapitre 1). Ceci étant dit, MarieClaire Patron avait pour objectif de nous offrir là quelques éléments de compréhension d'un problème très actuel aux conséquences encore peu mesurées et qui pourraient menacer la compétitivité de l'économie française si les responsables politiques ne le prennent pas en compte plus sérieusement et ce, dans un avenir proche. En effet, dans un contexte économique extrêmement fragile et hautement compétitif, la question de la gestion des jeunes diplômés requiert toute l'attention des pouvoirs publics. 
La problématique est que la France souffre des conséquences de l'âge avancé de sa population politique qui $d e$ facto engendre un conservatisme plus important dans les décisions et mesures prises. Cependant, les jeunes diplômés français d'aujourd'hui, au contraire de leurs prédécesseurs, n'attendront pas que la classe politique se réveille et prenne leurs problèmes au sérieux. Ils s'en iront offrir leurs compétences ailleurs et, ce faisant, enrichiront d'autres pays.

Cet ouvrage est publié au moment où la question du cumul des mandats politiques liée à celle de l'âge moyen des hommes politiques français est remise sur le devant de la scène (Le Monde, 2012). La compétition se déroule non seulement au niveau national, mais aussi au niveau international en matière de compétitivité. Comme le soulève l'auteure, le système social français se voit menacé par ce mouvement migratoire, car c'est justement la classe moyenne qui fournit le plus gros effort en termes d'impôts (chapitre 2).

En dépassant le cadre national, MarieClaire Patron apporte une vision singulière et une analyse personnelle d'une situation bien particulière. Elle met implicitement en concurrence des systèmes étatiques ne pouvant finalement que très rarement être confrontés au niveau micro social, c'est-à-dire de situation individuelle à situation individuelle. L'auteure présente les arguments qui, soit découragent les diplômés français à continuer leur carrière dans leur pays, soit séduisent les plus ambitieux et téméraires d'entre eux à faire le grand saut pour s'offrir une carrière à l'étranger voire à l'international. La question qui traverse l'ouvrage en filigrane est de savoir si ces expatriations sont plutôt choisies ou si elles résultent de circonstances qui obligent les individus à s'expatrier. Les deux situations sont bien différentes et le lecteur prend conscience, au fil de l'ouvrage, de la complexité d'une telle thématique.
La diversité des profils et des expériences des enquêtés alimente la description en la ponctuant de témoignages poignants et explicites sur les raisons des choix effectués par les expatriés. Dépassant la subjectivité propre à toute étude réalisée par entretiens, les propos relatés nous permettent de saisir plus clairement les enjeux qui entourent ces mouvements de population. Marie-Claire Patron utilise un cadre théorique inspiré des travaux de Pierre Bourdieu, complété et enrichi par les études de Louis Chauvel, pour expliquer I'accroissement des expatriations des Français (chapitre 3). Elle a cherché à resituer son étude dans la perspective politique française, notamment en ajoutant des éléments des différents discours politiques énoncés depuis les années 1980 sur la question.

Marie-Claire Patron, se faisant l'écho des riches propos recueillis, énonce quelques pistes d'explication de l'expatriation de la population étudiée. Parmi elles, le lecteur trouvera le fait que la société française soit peu encline à encourager l'initiative et surtout, à récompenser les performances réalisées (chapitre 5), dues aux efforts individuels et non en résultat d'un héritage quel qu'il soit. Effectivement, les propos des enquêtés laissent transparaître une certaine amertume quant au décalage observé entre les efforts et les investissements réalisés, et leur reconnaissance par les institutions privées et publiques (chapitre 6) ; les enquêtés se sentent quelque peu étriqués dans leur société à l'issue de leur parcours pourtant subtilement construit. Ce sentiment n'est pas nouveau, il avait déjà été constaté par Henri Mendras lors de son passage à Chicago (Mendras, 1999) et Michel Foucault en Suède et auxÉtats-Unis dans les années 1970 (Gros, 2010). L'expatrié français se heurte, à son retour, à l'incompréhension et au rejet dont il est la victime. Non seulement, il vient de vivre une expérience, certes enrichissante, mais douloureuse et de plus, il se voit jalousé et nié en raison de celle-ci. II ne s'agit pas ici de démontrer la 
faible considération d'une année d'études à l'étranger par les universités françaises, mais bien d'expliquer que corroborée à un manque de débouchés sur le marché du travail national, les diplômés français perçoivent, finalement assez mal, les fruits de leurs investissements (chapitres 2 et 5).

La rigidité du système corrélée à la frustration des enquêtés dans leurs démarches de recherche d'emploi les poussent alors à envisager un nouveau départ, qui résoudrait semble-t-il un besoin d'engagement dans une situation professionnelle. Parmi les enquêtés expatriés, le partage d'une forte réticence à un éventuel retour en France est à remarquer. En faisant I'unanimité, ce sentiment montre que ces Français ont finalement trouvé mieux ailleurs, sans regret, et c'est certainement le point le plus préoccupant (chapitres 6 et 7).

Cette lecture sera à compléter avec des études supplémentaires spécialisées sur telles ou telles populations pour pouvoir affiner la problématique proposée par Marie-Claire Patron et notamment en ce qui concerne les attentes des diplômés dans un contexte économique et social dans lequel elles sont formulées. De plus, il est un axe de réflexion qui n'est pas abordé dans l'ouvrage et qui pourtant pourrait venir compléter le questionnement. Qu'en est-il des étrangers diplômés de l'enseignement supérieur ayant émigré vers la France il y a quelques années et qui retournent vers leurs pays d'origine? S'interroger sur les raisons d'un tel revirement pourrait permettre de mettre en lumière les reproches formulés par les enquêtés quant à leur non désir de revenir en France.
Cet ouvrage apporte un éclairage concret sur un sujet fortement théorisé, mais peu étudié empiriquement, notamment depuis la crise financière et les propositions politiques quant à la régulation de l'immigration et de l'émigration en France. II a aussi le mérite de combler un manque de connaissances sérieuses et de proposer des explications sur un processus pourtant connu, celui souvent qualifié de " fuite des cerveaux ".

\section{Références bibliographiques}

Gros Frédéric (2010 [1996]) Michel Foucault, Paris, Que sais-je ?, 127 p.

Le Monde (2012) Le Monde, 10 novembre 2012, pp. 2-3

Mendras Henri (1999) Comment devenir sociologue? Souvenirs d'un vieux mandarin, Paris, Actes Sud, $334 \mathrm{p}$.

Patron Marie-Claire (2009) Diary of a French Girl. Surviving Intercultural Encounters, Robina, Bond University Press, $215 \mathrm{p}$.

Patron Marie-Claire (2007) Culture and Identity in Study Abroad Contexts: After Australia French without France, Bern, Peter Lang AG, International Academic Publishers, $332 \mathrm{p}$.

Philip Françoise (2007) La mobilité intra-européenne comme vecteur structurant à une appartenance supra-nationale. Approche sociologique de cette " multiterritorialisation complexe ", The Romanian Journal of European Studies, 5-6, pp. 33-43.

Pascaline Lorentz Docteure en Sociologie Université de Strasbourg 